\title{
La santé publique, un acteur majeur des politiques urbaines de transport actif?
}

Carole Clavier, France Gagnon, Sophie Paquin, Katie Hayes, Blake Poland, Beth Savan et Nina Escoute

\section{CpenEdition}

Journals

Édition électronique

URL : http://journals.openedition.org/rfst/330

DOI : $10.4000 /$ rfst.330

ISSN : 2492-3672

Éditeur

Espaces et SOciétés (UMR 6590)

\section{Référence électronique}

Carole Clavier, France Gagnon, Sophie Paquin, Katie Hayes, Blake Poland, Beth Savan et Nina Escoute, "La santé publique, un acteur majeur des politiques urbaines de transport actif? 》, Revue francophone sur la santé et les territoires [En ligne], Mobilités Transports et Santé, mis en ligne le 27 mai 2019, consulté le 06 avril 2021. URL : http://journals.openedition.org/rfst/330 ; DOI : https://doi.org/ $10.4000 /$ rfst.330

Ce document a été généré automatiquement le 6 avril 2021.

La Revue francophone sur la santé et les territoires est mise à disposition selon les termes de la Licence Creative Commons Attribution - Pas d'Utilisation Commerciale - Partage dans les Mêmes Conditions 4.0 International. 


\title{
La santé publique, un acteur majeur des politiques urbaines de transport actif?
}

\author{
Carole Clavier, France Gagnon, Sophie Paquin, Katie Hayes, Blake Poland, \\ Beth Savan et Nina Escoute
}

Cette recherche a bénéficié d'un financement de la Fondation des Maladies du Cour et de l'AVC dans le cadre du concours des Instituts de Recherche en Santé du Canada - Subvention de recherche interventionnelle en santé des populations (2014-2018, C. Clavier et F. Gagnon, chercheures principales). La recherche a été approuvée par les comités d'éthique de la recherche de l'UQAM (\#S-703818-66), de l'Université de Toronto (\#31140) et de Toronto Public Health (\#2015-06). Nous remercions Carl Marquis-Chouinard et Daniel Arancibia, assistants de recherche pour le projet respectivement à l'UQAM et à l'Université de Toronto.

\section{Introduction}

1 Les politiques urbaines qui encouragent les déplacements actifs à pied et à vélo sont réputées être favorables à la santé de la population (Paquin \& Dubé, 2011). Leurs bienfaits découlent d'une augmentation de la pratique de l'activité physique et de ses conséquences en termes de réduction des risques liés à l'obésité, aux maladies cardiovasculaires, aux maladies chroniques et à la dépression (Litman, 2003 ; Reynolds, 2010), d'une amélioration de la qualité de l'air et du bruit associés à la circulation automobile (Giles-Corti, Foster, Shilton, \& Falconer, 2010) et d'une amélioration des environnements bâtis, qui deviennent plus conviviaux, plus denses et aménagés pour réduire les risques de blessures et de décès liés aux transports actifs (Frank, Giles-Corti, \& Ewing, 2016; Reynolds, 2010). Pourtant, l'action sur ces déterminants sociaux de la santé (Marmot \& Wilkinson, 2006) échappe en grande partie à la santé publique : créer des infrastructures cyclables ou piétonnes (élargissement des trottoirs, pistes cyclables, etc.), améliorer l'accès aux transports en commun, modifier la réglementation pour réduire la vitesse de circulation des automobiles sont autant d'interventions qui 
relèvent de la responsabilité des secteurs des transports ou de l'aménagement urbain, et non des acteurs de santé publique.

2 En d'autres termes, créer des politiques favorables à la santé implique de créer des liens avec les acteurs de ces autres secteurs d'action publique. Ce faisant, les acteurs de santé publique risquent de se heurter « aux jeux politiques [politics] et de pouvoir » (de Leeuw, 2017, p. 344). L'objectif de cet article est d'étudier comment, sur le terrain, les acteurs de santé publique - en particulier les autorités régionales et municipales de santé publique - prennent part à l'action publique pour intégrer leurs préoccupations de santé dans les politiques urbaines. Puisque les leviers d'action relèvent d'autres secteurs, comment interviennent-ils? Leurs actions peuvent-elles aller au-delà des plaidoyers et d'un effort de conviction? Et comment cet effort de conviction peut-il aboutir alors que la santé n'est pas l'objet premier de ces autres secteurs? Pour explorer ces enjeux, nous étudions le rôle des acteurs de santé publique dans le processus qui conduit deux villes, Montréal et Toronto, à inscrire à leur agenda (l'émergence) et à mettre en œuvre des politiques de transport actif, qui vont jusqu'à inclure des initiatives spécifiques de transformations de l'environnement bâti (la mise en œuvre). Cette étude de processus locaux est ancrée dans une théorie du processus de politiques publiques - le cadre des coalitions de cause (Sabatier \& Weible, 2007) - qui porte l'attention sur les interactions d'acteurs et sur les croyances et valeurs des acteurs.

3 Pour répondre à ces questions, nous présenterons d'abord le cadre conceptuel et la méthode de recherche. La présentation des résultats est organisée en trois parties centrées sur 1) la place de la santé dans les arguments de légitimation des politiques de transport actif ; 2) le rôle des acteurs de santé publique dans les processus d'émergence des politiques de transport actif; et 3) l'absence des acteurs de santé publique dans la mise en œuvre des initiatives spécifiques à l'étude. Ce dernier résultat contraste avec la forte présence des acteurs de santé publique et de leurs arguments dans l'émergence des politiques de transport actif à l'échelle de la ville. Nous discuterons ce paradoxe à la lumière des résultats contextuels dans la discussion.

\section{Cadre conceptuel}

4 Cet article s'inscrit dans une recherche comparative sur les politiques de transport actif à Montréal et Toronto, entendues comme des politiques potentiellement favorables à la santé. Dans cet article, nous interrogeons spécifiquement la contribution des acteurs de santé publique lors de l'émergence et de la mise en œuvre des politiques de transport actif.

5 Le premier élément à clarifier est la distinction entre ces deux étapes du processus de politiques publiques. L'émergence couvre le processus de construction d'un problème en problème qui mérite l'intérêt des autorités publiques, jusqu'à la prise d'une décision d'agir - par exemple, l'élaboration d'un plan ou d'un programme. La mise en œuvre correspond au choix et à la définition des modalités d'action, à l'attribution des ressources pour les réaliser et à la concrétisation de ces modalités d'action. Dans le cas des politiques de transport actif, la mise en œuvre ne correspond donc pas seulement aux travaux d'infrastructure pour implanter une piste cyclable, mais aussi au choix de la rue qui l'accueillera et à la définition des caractéristiques techniques de la piste cyclable. Précisons que nous ne postulons pas que la mise en œuvre découle 
logiquement de l'émergence des politiques publiques. Au contraire, la littérature a montré que le processus de politiques publiques est souvent chaotique : l'intervention de nouveaux acteurs ou la réinterprétation du problème peuvent conduire à des décalages entre émergence et mise en œuvre des politiques publiques (DeLeon, 1999; Hassenteufel, 2011).

6 Le second élément à retenir est notre intérêt pour le cadre des coalitions de cause, ou Advocacy Coalition Framework - ACF (Sabatier \& Weible, 2007; Weible et al., 2011). Ce dernier invite à considérer les interactions entre les acteurs du sous-système d'une politique publique, c'est-à-dire tous les acteurs potentiellement concernés par le transport actif qu'ils soient élus, fonctionnaires, représentants de groupes d'intérêt, journalistes ou universitaires. Selon cette théorie, les acteurs qui partagent un ensemble de croyances quant au problème et aux solutions à adopter pour y remédier interagissent pour former des coalitions de cause, formelles ou informelles, dont l'objectif est d'influencer la décision publique dans le sens de leurs croyances. Les acteurs d'une même coalition peuvent appartenir à différentes organisations, aux intérêts souvent divergents et dont les interactions sont affectées par des rapports de pouvoir résultant du décalage entre leurs ressources, leur capacité d'action, leur légitimité, etc. Le changement de politique publique s'expliquerait donc par la capacité d'une nouvelle coalition à imposer son système de croyance (valeurs) ou par l'évolution des croyances au sein de la coalition dominante. En outre, les interactions entre les acteurs du sous-système sont influencées par le contexte dans lequel elles se déroulent. Il s'agit, d'une part, des paramètres relativement stables du sous-système (les structures constitutionnelles, les caractéristiques de l'environnement naturel et bâti, etc.) et, d'autre part, des événements externes au sous-système tels que les changements de l'opinion publique ou de gouvernements.

7 Pour les besoins de cet article, nous ne cherchons pas à établir l'existence de coalitions de causes opposées dans le sous-système du transport actif, ni à établir l'effectivité d'une coalition à influencer le changement de la politique du transport actif. De façon plus circonscrite, le choix de l'ACF signifie que nous portons l'attention sur les interactions entre les acteurs du sous-système (qui travaille avec qui), sur la façon dont ils cadrent le problème du transport actif et sur les éléments contextuels.

\section{Méthode}

8 Notre stratégie de recherche repose sur la comparaison de deux cas similaires au regard de l'objet de recherche, Montréal (Québec, Canada) et Toronto (Ontario, Canada). Choisir des cas qui présentent des similarités permet de comparer des processus de politiques publiques complexes, qui font intervenir une multiplicité d'acteurs publics, privés et communautaires, tout en limitant les facteurs de variation entre chaque cas (Sartori, 1991). La comparaison est donc centrée sur les interactions entre les acteurs à Montréal et Toronto. Ces deux grandes municipalités urbaines partagent un intérêt pour les politiques de transport actif depuis au moins le début des années 2000 ; toutes deux font face à des difficultés de mise en œuvre de ces politiques et, enfin, toutes deux avaient initié la réalisation de bilans ou de processus de révision de leurs plans d'aménagement ou de transport au moment de débuter cette recherche (2013). La proportion des déplacements actifs vers le travail est similaire dans les deux villes selon le recensement de 2016, soit 7,2\% à Montréal et 6,7\% à Toronto (Statistics 
Canada, 2017). Avec 5,2\% des déplacements effectués à pied dans chaque ville, la différence est attribuable à une proportion légèrement plus élevée de déplacements à vélo à Montréal ( $2 \%$ contre $1,4 \%$ à Toronto). Ces chiffres dénotent une légère augmentation des déplacements vers le travail à vélo à Montréal depuis 2011, mais une baisse des déplacements à pied à Toronto depuis 2006 (7\%). Ils masquent également des variations dans chaque ville, puisque les déplacements actifs sont plus importants dans les zones denses du centre-ville (Toronto Cycling, 2012 ; Vélo Québec, 2015). Le réseau cyclable sur route s'est étendu dans les deux villes au cours de la dernière décennie, passant de 400 à $746 \mathrm{~km}$ de 2008 à 2015 à Montréal (Vélo Québec, 2015), et de 563 à $619 \mathrm{~km}$ de 2013 à 2019 à Toronto (City of Toronto, 2019).

9 Malgré ces caractéristiques similaires, les deux villes diffèrent par leur taille (1,7 million d'habitants à Montréal et 2,8 millions à Toronto), leur superficie $\left(431 \mathrm{~km}^{2}\right.$ à Montréal et $630 \mathrm{~km} 2$ à Toronto) et leur organisation politique (figures 1 et 2). En effet, les fusions municipales des années 2000 ont étendu la ville de Toronto en lui adjoignant les municipalités voisines de Etobicoke, York, North York, East York et Scarborough, modifiant notamment l'équilibre politique entre la ville-centre très densément peuplée et les banlieues (Horak, 2012). À Montréal, une des conséquences des fusions et défusions municipales des années 2000 est la reconnaissance des arrondissements et de leurs élus dans la gouvernance municipale (Boudreau, Hamel, Jouve, \& Keil, 2006). En raison du partage de compétences entre la ville-centre et les arrondissements, on observe une grande variabilité des interventions en faveur du transport actif d'un arrondissement à l'autre. La comparaison inclut également un facteur intéressant de distinction du point de vue de l'organisation administrative, puisque la santé publique est de juridiction provinciale au Québec et municipale en Ontario. La comparaison permet donc de questionner l'influence de ce positionnement administratif sur la capacité des acteurs de santé publique à influencer les politiques municipales de transport actif. 
Figure 1 : Toronto (Ontario), Canada

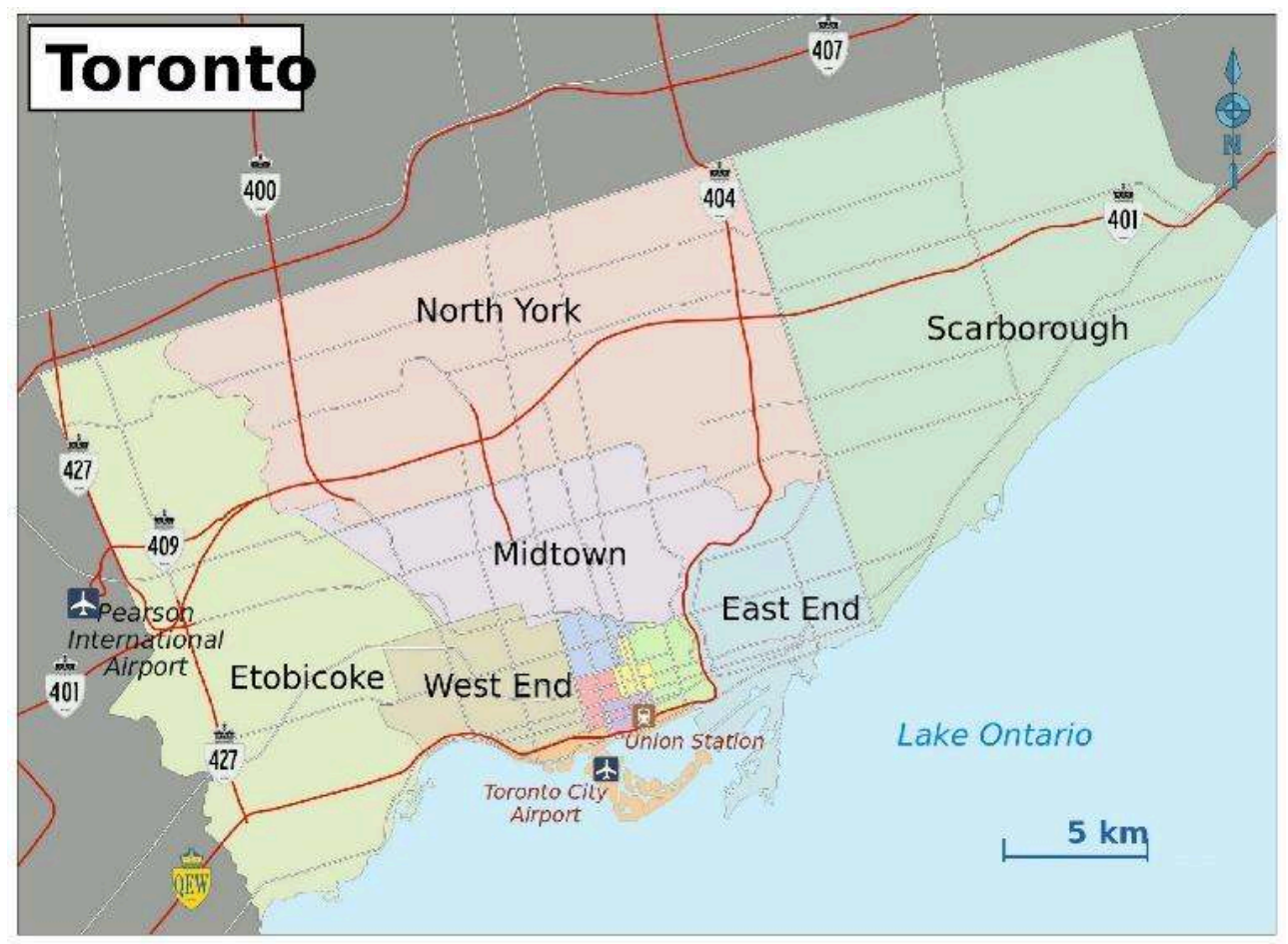

(Source : By Lencer - own work Used : OpenStreetMap http://www.toronto.ca/demographics/ profiles_map_and_index.htm Mini map created with Greater toronto area map-de.svg by NordNordWest, CC BY-SA 3.0, https://commons. wikimedia.org/w/index. php?curid=6077610 ) 


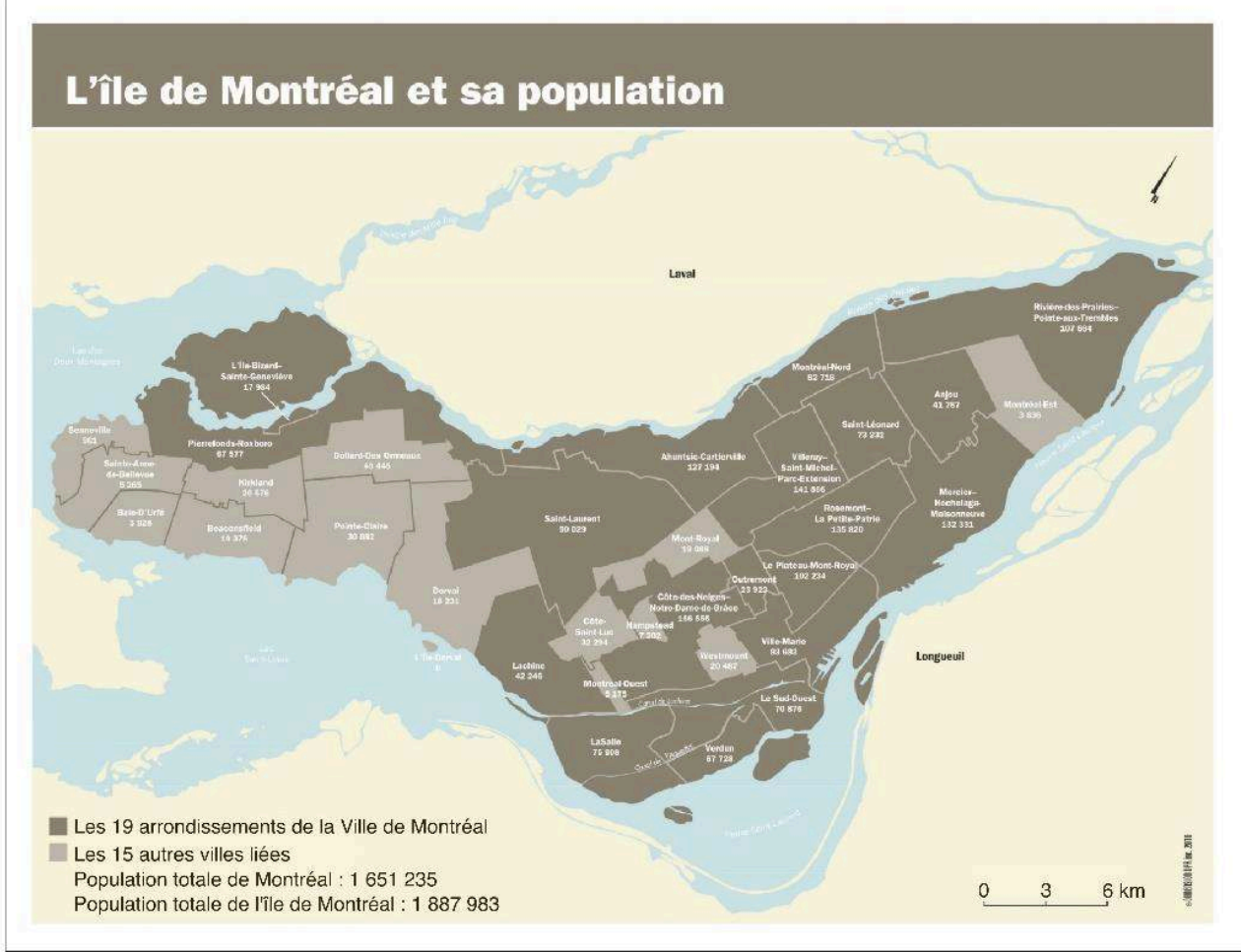

Source : http://ville.montreal.qc.ca/pls/portal/docs/page/prt_vdm_fr/media/documents/ CARTE_ILEMONTREALARRON.pdf

Pour étudier tant l'émergence que la mise en œuvre des politiques de transport actif, nous avons ajouté un deuxième niveau à la comparaison, en ciblant deux initiatives spécifiques dans chaque ville qui contribuent à concrétiser les objectifs de la politique de transport actif. Ainsi, la comparaison a porté sur trois moments clés : la mise à l'agenda initiale de la question du transport actif à partir du début des années 2000 jusqu'en 2015-2016, au moment de réaliser les entretiens; de la proposition jusqu'à l'adoption (2012-2013) de deux initiatives spécifiques de transport actif dans chaque ville ; la mise en œuvre de ces deux initiatives depuis leur adoption jusqu'à 2015-2016. La comparabilité des initiatives étudiées repose sur deux caractéristiques: 1) considérant l'importance des caractéristiques de l'environnement bâti pour le développement et la pratique du transport actif (Cervero, Sarmiento, Jacoby, Gomez, \& Neiman, 2009; Feuillet et al., 2016; Frank et al., 2016), elles proposent des transformations physiques de secteurs urbains; 2) la mise en œuvre des initiatives prend en compte les déplacements à pied et à vélo, quelles que soient les raisons qui ont mené à leur développement. Bien qu'elles diffèrent par l'ampleur des changements apportés à l'environnement bâti, deux initiatives proposent le réaménagement d'une rue, incluant la création de voies cyclables (intersection Saint-Laurent Bellechasse à Montréal et piste cyclable à contresens de la rue Shaw à Toronto) et les deux autres intègrent le transport actif dans le cadre du réaménagement d'un quartier ou d'une rue commerçante (Quartier vert Parc-Extension à Montréal et revitalisation de la rue Roncesvalle à Toronto).

11 Nous avons d'abord procédé à une analyse de données documentaires: les plans et programmes municipaux de transport et d'urbanisme qui exposent les orientations 
officielles en matière de transport actif; les documents (rapports, mémoires de consultations) produits par les acteurs de santé publique, par les groupes d'intérêt cyclistes et piétons ainsi que par des groupes environnementaux qui intègrent le transport actif parmi leurs préoccupations. Enfin, nous avons procédé à des entretiens semi-directifs avec les principaux acteurs (Montréal $n=20$; Toronto $n=20$ ) des soussystèmes du transport actif et des initiatives étudiées dans chaque ville. Ces entretiens ont fait l'objet d'un codage thématique sur une plateforme d'analyse qualitative en ligne, Dedoose, facilitant la coordination entre les membres de l'équipe situés à Montréal, Québec et Toronto. Le codage thématique suivait les trois moments clés ainsi que les grandes catégories conceptuelles de l'ACF (les acteurs du sous-système, leurs interactions, les paramètres relativement stables et les évènements externes).

L'analyse des différentes sources de données a permis de reconstituer le sous-système du transport actif dans chacun des cas et les variations de la dynamique du soussystème entre les moments à l'étude (émergence et mise en œuvre).

Pour les besoins de cet article, nous mettons l'accent sur les acteurs de santé publique, dont les autorités régionales et municipales de santé publique. Il s'agit de la direction de santé publique de Montréal (DSP de Montréal), une administration déconcentrée du réseau provincial de la santé et des services sociaux, et Toronto Public Health, une direction de l'administration municipale de la ville de Toronto. Nous prenons également en considération le rôle d'autres acteurs de santé publique qui interviennent dans les sous-systèmes des politiques de transport actif, en particulier ceux provenant d'organismes publics tels que les ministères de santé ou l'Agence de santé publique du Canada (ASPC), d'établissements universitaires et d'organisations nongouvernementales qui ont une mission de santé publique. En plus de considérer le rôle des acteurs, l'ACF porte l'attention sur les croyances véhiculées par les acteurs à propos de la politique publique (les valeurs). Concrètement, nous souhaitons donc retracer les interactions auxquelles participent les acteurs de santé publique et la façon dont les connaissances et les valeurs de santé sont mobilisées dans les discours des acteurs du sous-système, tant lors de l'émergence que de la mise en œuvre des politiques de transport actif.

\section{La santé, un argument de légitimation de l'émergence des politiques de transport actif}

Tant à Montréal qu'à Toronto, la santé est très présente dans les débats et processus qui président à l'émergence des politiques de transport actif. Dans cette section, qui correspond au premier moment de la comparaison, nous verrons comment le transport actif est devenu une préoccupation pour les acteurs de santé publique. Bien qu'il soit difficile d'établir un lien de causalité, nous constaterons également que la santé est une valeur mise de l'avant dans les documents officiels et les discours de légitimation des mesures favorables au transport actif, y compris par des acteurs d'autres secteurs. Enfin, nous considérerons la sécurité des déplacements actifs, qui semble être le point focal de l'intérêt des différents acteurs locaux. 


\section{La construction d'un argumentaire de santé en faveur du transport actif}

15 À Montréal comme à Toronto, les acteurs de santé publique ont progressivement construit leur argumentaire sur la nécessité d'une politique de transport actif. Dans les deux cas, ces efforts remontent jusque dans les années 1990, avec l'émergence d'une préoccupation pour la qualité de l'air en ville. La construction de ces argumentaires a culminé avec la publication de rapports et études sur les liens entre transport et santé qui sont devenus des points de référence dans le processus d'émergence des politiques de transport actif. Nous les qualifions de points de référence car ces publications ont été mentionnées à plusieurs reprises par les autres acteurs du sous-système de la politique de transport actif dans leurs villes respectives.

À la fin des années 1990, la DSP de Montréal mène, à la demande de la ville de Montréal, des travaux sur la pollution de l'air, en particulier sur les particules fines produites par la circulation automobile, ainsi qu'une consultation auprès des élus de la communauté métropolitaine de Montréal sur leurs perceptions des enjeux de transport, d'environnement et de santé publique (smog). Ces travaux, poursuivis dans les années suivantes, établissent un ensemble de liens entre le transport - en particulier automobile - et la santé :

« Fin des années 1990, début 2000, on commence à considérer qu'il y a beaucoup de problèmes de santé associés au transport comme tel, principalement les blessés de la route, mais aussi les maladies respiratoires associées à la pollution, et aussi, évidemment, les maladies liées à l'inactivité physique, cardiovasculaires, cancer. À partir des années fin 1990, mais surtout début 2000, on commence à reconnaître que ce sont des problèmes de santé associés au système de transport » (Montréal, acteur de santé publique).

17 En 2006, la DSP de Montréal se positionne formellement quant à la politique de transport avec la parution du rapport sur la santé de la population montréalaise intitulé Le transport urbain, une question de santé (Direction de santé publique, 2006). Produit en consultation avec des universitaires et avec l'appui de réseaux internationaux de recherche, le rapport dresse un état des connaissances sur les liens entre santé, transport et environnement bâti, explorant notamment les liens entre pollution et santé, le bilan de sécurité routière, le transport actif et la sédentarité, le transport et l'exclusion sociale. Il se conclut par des propositions d'action pour réduire la dépendance à l'automobile, accroître le recours au transport en commun et aux déplacements actifs et la mobilisation des acteurs locaux et municipaux. Plusieurs acteurs rencontrés lors de notre recherche le considèrent comme un document de référence qui leur permet de mobiliser des arguments scientifiques en appui à leurs propositions en faveur du transport actif. La publication du rapport est aussi considérée comme un moment charnière, car sa diffusion a contribué au débat public et médiatique sur le transport actif pendant la période de préparation du Plan de transport de la ville paru moins de deux ans plus tard. À la même période, l'Institut national de santé publique du Québec (INSPQ) publie déjà des rapports sur les aménagements favorables à l'activité physique en général et au transport actif en particulier, contribuant ainsi à la mise à l'agenda du transport actif dans les villes.

18 La qualité de l'air est également l'un des vecteurs de l'émergence de la préoccupation pour le transport et la santé à Toronto dans les années 1990 : 
« We came at it through Air Quality, we did a big air quality study, and that was in 1993 [...] Air pollution Burden of Illness in Toronto 2004 got a lot of media attention and again transportation sector, among other sources. This one was interesting, 2004, Agenda for Action on Air and Health, a bit of a road map for the Provincial Government to do more, whether it's fuel emission standards, whether again it's industrial pollution or transportation. So again, flagging the transportation thing » (Toronto, acteur de santé publique)i.

Ces premiers travaux ont mené, au début des années 2010, à une réflexion d'ensemble sur les liens entre l'environnement bâti et la santé dans lesquels le transport est identifié comme un déterminant de la santé. À la même époque, Toronto Public Health - tout comme la DSP de Montréal - s'est engagée dans un partenariat de recherche sur la santé et l'environnement bâti, financé dans le cadre d'une initiative du Partenariat canadien contre le cancer. Dans ce contexte, entre 2011 et 2014, Toronto Public Health a publié une série de rapports établissant les liens entre la santé et l'environnement bâti, et proposant des stratégies d'action pour encourager et faciliter les déplacements à pied et à vélo (Toronto Public Health, 2011, 2012, 2013, 2014). Parmi ces publications, le rapport Road to Health: Improving walking and cycling in Toronto était dédié à des propositions pour développer et sécuriser les déplacements à pied et à vélo. L'une d'entre elles consistait à réduire la vitesse de circulation à $30 \mathrm{~km} / \mathrm{h}$ dans les rues résidentielles.

\section{Des arguments de légitimation du transport actif influencés par la santé}

Bien qu'il soit difficile d'établir un lien de causalité direct entre les données et les argumentaires produits par les acteurs de santé publique et les documents officiels de politique de transport actif, nous constatons que ces derniers incluent des arguments qui soulignent les bénéfices du transport actif pour la santé de la population. En 2008, la ville de Montréal s'est dotée d'un Plan de transport dont un chapitre est consacré aux «modes actifs» (Ville de Montréal, 2008). Parmi les actions proposées, retenons l'augmentation du réseau cyclable, la sécurité des piétons et la mise en œuvre de la Charte du piéton. Cette dernière, élaborée en 2006, est présentée comme un des éléments de positionnement du Plan de transport. Elle affirme que sécuriser les déplacements à pied contribue à améliorer la qualité de vie et la santé des citoyens. On observe un engagement similaire de la part des autorités municipales de Toronto. Tout d'abord, en mai 2002, une conseillère municipale et l'urbaniste Jane Jacobs - connue notamment pour son ouvrage Déclin et survie des grandes villes américaines (1961) demandent à la ville de Toronto d'adopter une Charte du piéton visant à rendre les déplacements à pied plus sécuritaires et plus accessibles pour l'ensemble de la population. La Charte priorise six principes dont ceux de sécurité et de santé et bienêtre et affirme que «un environnement urbain qui encourage et facilite la marche soutient la santé, la vitalité et la sécurité de la communauté » (City of Toronto, 2002). Le Toronto Bike Plan (City of Toronto, 2001), intégré dans le Official Plan de la ville en 2006, mettait déjà l'accent sur la sécurisation des déplacements pour réduire le nombre de collisions et de blessures. Le document Toronto Walking Strategy justifie les transformations des infrastructures piétonnes notamment en référence aux bienfaits pour la santé, citant les effets de la pollution de l'air sur la mortalité prématurée et la sédentarité croissante des adultes (City of Toronto, 2009, p. 6-7). À la suite de la révision 
du chapitre Shaping the City du Official Plan de la ville en 2014, la référence aux bienfaits pour la santé est la première justification avancée pour soutenir les modifications aux infrastructures pédestres et cyclistes de la ville (City of Toronto, 2014, p. 2-27).

Nos résultats montrent également que les acteurs d'autres secteurs reconnaissent l'impulsion que les travaux de la santé publique ont donnée à la réflexion et à l'action collective sur les politiques de transport actif, à l'instar de ce représentant de groupe cycliste à Toronto :

«I think that the emergence of Public Health into this area has been a huge boost in terms of the credibility and the reach of the importance of active transportation " (Toronto, représentant d'un groupe d'intérêt en transport actif).

De même, un conseiller municipal de Toronto souligne l'importance des publications de la santé publique mettant en lien la sédentarité, l'environnement bâti et la santé, tandis qu'un fonctionnaire de la direction des transports explique que les recherches en santé ont apporté un angle différent à l'enjeu du transport actif, créant à la fois des tensions et de nouvelles pistes de réflexion. D'ailleurs, plusieurs entretiens mentionnent que l'urbaniste en chef de la ville de Toronto était l'une des actrices qui, sans nécessairement les formuler en termes de santé, a contribué à faire avancer des propositions des acteurs de santé publique telles que les "rues complètes " (Complete Streets). De plus, certains acteurs vont jusqu'à reprendre à leur compte des arguments scientifiques associant la santé et le transport actif. À Toronto, l'énoncé de mission actuel de l'organisme non-gouvernemental Toronto Center for Active Transportation (financé en partie par l'Agence de santé publique du Canada) est centré sur la santé, décrivant la « vision de villes dynamiques où l'air est pur, la population en santé et le système de transport privilégie la marche et le vélo ». À Montréal, ce représentant d'un groupe d'intérêt environnemental considère que l'argument santé est plus apte que d'autres à convaincre les citoyens de changer leurs modes de déplacements :

«Et puis, le volet, on va dire, aussi santé, qui est en ce moment le principal axe de conviction, on peut dire, des gens, les gens prennent conscience des modes de vie sédentaires comme étant obésogènes. Les gens ont envie de faire du sport, ont envie de se tenir en santé et privilégient donc des modes de transport qui leur permettent d'être plus en forme.

$\mathrm{Q}$ : Ça vaut dire que l'axe santé ou les bienfaits du déplacement à pied ou à vélo pour la santé, pour vous, ça fait partie des arguments que vous mettez de l'avant?

$\mathrm{R}$ : Le principal, je dirais. Après, on essaie beaucoup de parler d'économique, mais les gens, en tout cas les automobilistes, n'en tiennent pas vraiment compte.» (Montréal, représentant d'un groupe d'intérêt environnemental).

Alors que cet extrait renvoie à l'aspect bien-être de la santé, c'est bien l'argument de la sécurité des déplacements que les acteurs des autres secteurs s'approprient le plus fréquemment.

\section{La sécurité, principale dimension de la santé qui mobilise les autres acteurs locaux}

Tous les acteurs rencontrés insistent sur un point: le développement de politiques de transport actif est nécessaire pour améliorer les infrastructures et sécuriser les déplacements à pied et à vélo. Ainsi :

«C'est parti d'une analyse de la sécurité piétons et cyclistes sur [les principales rues de cette zone]. Ça, c'était en 2006. ... C'était vraiment pour que les travailleurs et les gens puissent se déplacer de façon sécuritaire, mais aussi de façon à diminuer aussi 
le transport en automobile dans le quartier » (Montréal, représentant d'un groupe d'intérêt environnemental)

"A few of us came together and we started an organization called Advocacy for Respect for Cyclists. And one of the things that we were calling for was a coroner's inquest into traffic, into the fatalities that were happening with large trucks. And that was... we were able to convince the coroner to do that and there was a fair amount of attention at the time paid to the issue " (Toronto, représentant d'un groupe d'intérêt en transport actif).

focal de l'enjeu de sécurisation des déplacements, particulièrement après le décès d'une cycliste lors d'une collision avec un camion sous un viaduc en 2014. En outre, plusieurs de nos interlocuteurs ont fait référence à une étude, dirigée par un médecin de la DSP de Montréal, sur la répartition géographique et la prévalence des collisions avec blessés à Montréal (Morency et al., 2013). Les cartes de cette recherche ont contribué au débat sur la sécurité des piétons en fournissant des données jusqu'ici inexistantes. Elles ont trouvé un écho concret auprès des citoyens et des groupes favorables au transport actif, car elles montrent visuellement la dangerosité des intersections pour les piétons. Diffusées largement, ces données permettent aux groupes et aux citoyens d'objectiver leurs perceptions du risque de collision dans les rues de la ville et d'appuyer leurs demandes d'aménagements plus sécuritaires pour la pratique de la marche et du vélo.

\section{Les acteurs de santé publique en interaction lors de l'émergence des politiques de transport actif}

Selon le modèle de l'ACF, les acteurs du sous-système interagissent sur la base de leurs visions communes du problème et des solutions à mettre de l'avant. Dans cette section, qui correspond toujours au premier moment de la comparaison, nous nous intéresserons donc aux interactions entre les acteurs de santé publique et les autres acteurs du sous-système afin de comprendre comment les argumentaires de santé en faveur du transport actif y circulent. Les acteurs de santé publique orientent leurs interactions en faveur des politiques de transport actif dans deux directions complémentaires, soit d'abord envers les élus et les fonctionnaires municipaux et, ensuite, envers les autres groupes d'intérêt et institutions qui partagent l'objectif du 
développement de politiques de transport actif. Ces échanges s'inscrivent dans des réseaux de collaborations croisées dans lesquels chaque acteur (santé publique, groupes cyclistes, piétons ou environnementaux, autres institutions) travaille à la promotion de ses propres objectifs et intérêts en développant un réseau de partenaires financiers, intellectuels et d'action. Les liens que nous avons retracés dans cette section ne représentent donc qu'une partie des liens réels entre tous les acteurs.

\section{Interactions avec les élus et fonctionnaires municipaux}

Tout d'abord, les acteurs de santé publique cherchent à interagir avec les élus et les fonctionnaires municipaux, c'est-à-dire avec les acteurs qui détiennent la capacité d'action pour modifier l'environnement bâti dans un sens favorable aux déplacements à pied et à vélo. À Montréal, la DSP maintient des liens formels avec la ville par la participation à différents comités de travail et par le dépôt de mémoires lors de consultations publiques à propos de transformations majeures de l'environnement bâti (ex. : le réaménagement du super échangeur Turcot en milieu densément peuplé ou les projets de piétonisation de certaines rues). À cela s'ajoutent des représentations auprès d'élus ou de fonctionnaires aux différentes échelles de gouvernement concernées afin d'expliquer les impacts sanitaires des projets discutés. Bien que, dans le passé, les deux institutions aient déjà collaboré, ceci semble plus laborieux dans la période récente. Plusieurs acteurs ont souligné la difficulté d'établir des collaborations durables avec la direction des transports de la ville et parfois la difficulté d'accéder aux fonctionnaires. Souvent, les échanges semblent se dérouler sur une base individuelle, entre collègues de différentes organisations, mais partageant des intérêts professionnels similaires. Ainsi, un acteur de santé publique relate l'absence de travail conjoint avec l'administration municipale et ses élus amenant son organisme à diffuser les données pour qu'elles soient utilisées en premier lieu par les organismes communautaires et les citoyens :

«Cette information-là a percolé à travers les groupes communautaires et dans beaucoup d'arrondissements [...]. Mais dans la ville centrale [...] on participait seulement aux consultations officielles de l'office de consultations publiques. On n'a jamais eu de processus de travail conjoint sur rien d'autre que des mémoires externes lors de processus de consultation de tous les citoyens. Il n'y a jamais eu rien d'autre. » (Montréal, acteur de santé publique)

Parmi les explications de ces difficultés de collaboration avancées dans les entretiens, retenons le fait que certaines données seraient perçues comme une remise en cause du travail des administrations municipales, ainsi que la faible efficacité du fonctionnement interne des administrations municipales, en particulier de la coordination entre les services et avec les élus.

À Toronto, on note le même type d'interactions à la différence toutefois que les acteurs de l'autorité locale de santé publique sont eux-mêmes des fonctionnaires municipaux. Bien que les acteurs de santé publique soient reconnus pour leur " push» (Toronto, chercheur universitaire) crucial pour l'émergence de la politique de transport actif, leurs interactions avec les élus ou fonctionnaires en charge des transports et de l'aménagement urbain ne semblent pas être plus fluides pour autant. Les intérêts des acteurs de santé publique ne coïncident pas nécessairement avec ceux des autres directions de l'administration municipale. Ainsi, en 2015, la proposition de Toronto Public Health de limiter la circulation automobile à $30 \mathrm{~km} / \mathrm{h}$ dans les quartiers 
résidentiels a suscité une controverse publique. En ligne avec ses convictions quant à l'importance de l'automobile dans l'espace urbain, le maire Rob Ford a qualifié la proposition de «nuts, nuts, nuts» et demandé «why does he [le directeur de santé publique] still have a job? », avant d'être contraint de s'excuser quelques mois plus tard (Dale, 2012). L'élu responsable du comité des travaux publics a également reproché publiquement au directeur de santé publique de se mêler d'un sujet pour lequel il ne serait pas compétent, lui conseillant «If he wants to lower speed limits, maybe he should apply for the general manager's job in the transportation department » (Dale, 2012). De son côté, le directeur de santé publique a défendu sa recommandation en référence à la science et soulignant qu'il revient aux élus de départager les intérêts en jeu et de prendre les décisions politiques (Rider, 2012). Pour certains de nos interlocuteurs, cette controverse a été perçue comme le signe d'attentes "irréalistes » (Toronto, employé de la direction municipale des transports) de la part des acteurs de santé publique ou de la nécessité de dialoguer pour faire converger les attentes des acteurs de santé publique et du transport.

31 Au-delà du cas particulier, cette controverse rappelle surtout que les acteurs de santé publique interviennent dans un espace politique dans lequel leurs valeurs et leurs intérêts sont en compétition avec d'autres valeurs et intérêts. En effet, les politiques de transport actif font intervenir des acteurs dont les préférences en matière de transport et de conception du rôle de la santé publique sont distinctes, soulignant la persistance du partage de responsabilité traditionnel entre les secteurs de l'action publique (Clavier \& Gagnon, 2013).

\section{Interactions avec les groupes concernés par le transport actif}

32 Les acteurs de santé publique recourent aussi à une stratégie d'élargissement du débat public pour faire évoluer les représentations du transport actif. Ils collaborent avec des acteurs non-gouvernementaux intéressés au développement de politiques de transport actif, tels que des groupes cyclistes, piétons ou environnementaux. Des collaborations avec des universitaires dont les recherches portent sur les systèmes de transport, la géographie des déplacements ou les liens entre activité physique et santé publique sont observées. Ces échanges s'inscrivent dans des logiques de construction d'alliances pour porter plus efficacement un message partagé, de co-construction de connaissances par la coopération à des projets de recherche, ainsi que dans des logiques d'action. Par exemple, la DSP de Montréal s'implique dans les comités scientifiques ou de suivi de certaines démarches d'aménagement urbain porté par des organismes publics ou associatifs. C'est le cas du comité scientifique du projet Quartier Vert Actif et en Santé mené par le Centre d'écologie urbaine de Montréal. Autre exemple, la DSP de Montréal détient un siège permanent sur le comité d'orientation de Montréal Métropole en santé et elle participe activement à la grande mobilisation partenariale publique-associative Montréal physiquement active rassemblant entre autres la ville de Montréal et une vingtaine d'organismes. Bien que le transport actif ne soit pas l'objet principal de ces forums, il fait partie des stratégies identifiées pour soutenir l'activité physique de la population.

33 À Toronto, les acteurs de santé publique ressortent également comme des partenaires de premier plan des groupes d'intérêt cyclistes et piétons. Leur contribution a permis d'élargir le débat sur le transport actif à des enjeux de santé des populations, incluant 
le bien-être des humains et des environnements sains. Le "big boost» (Toronto, représentant d'un groupe d'intérêt en transport actif) de la santé publique torontoise apporté aux groupes cyclistes et piétons repose là encore sur le développement de connaissances et sur leur diffusion, ainsi que sur le financement d'organismes nongouvernementaux en transport actif. Par exemple, le représentant d'un groupe cycliste et piéton souligne l'importance de tels partenariats pour l'action, en l'occurrence pour la promotion d'une nouvelle piste cyclable :

« [The Dundas East bike lane] is a great example to me of how there was a really good partnership between the community health organizations and the advocates and city staff to kind of work together to come up with a solution that resulted in a bike lane that wasn't in the bike plan, that no one quite believed it was going to happen, it meant taking away two lanes of traffic on a very kind of busy street. So I think that that was a really excellent example of the kind of advocacy that we need and the kind of partnerships that we need to make these things happen » (Toronto, représentant d'un groupe d'intérêt en transport actif)

Les acteurs locaux de santé publique entretiennent également des collaborations avec des universitaires, tant pour effectuer des recherches que pour intervenir au comité santé du conseil municipal en appui à des propositions de santé en faveur du transport actif. D'autres organismes comme l'Agence de santé publique du Canada et le Partenariat canadien contre le cancer financent aussi des organismes nongouvernementaux.

\section{La relative absence de la santé publique dans la mise en oeuvre des initiatives spécifiques de transport actif}

Dans cette section, nous considérons le rôle des acteurs de santé publique dans le contexte menant à chacune des initiatives spécifiques étudiées - c'est le deuxième moment de la comparaison - et dans la mise en œuvre de ces initiatives spécifiques - le troisième moment de la comparaison. La sécurité des déplacements à pied et à vélo est une préoccupation commune à toutes ces initiatives, mais, dans tous ces cas, les acteurs de santé publique eux-mêmes sont remarquablement absents des processus.

\section{La santé, parfois une préoccupation au départ des initiatives étudiées}

Les quatre initiatives spécifiques étudiées consistent en des projets de réaménagement de l'environnement bâti pour faciliter les déplacements à pied ou à vélo. Ces réaménagements répondent à des justifications variées, mais la seule qui est invoquée dans l'ensemble des cas est la sécurité des déplacements à pied et à vélo. L'initiative Quartier Vert Parc-Extension à Montréal est partie du constat des acteurs locaux que de nombreuses intersections étaient dangereuses, tant pour les piétons que pour les cyclistes. Elle participait de la démarche Quartier Vert Actif et en Santé qui repose sur la participation citoyenne pour repérer et proposer des aménagements de sécurité et de convivialité pour les déplacements actifs dans le quartier. La seconde initiative étudiée à Montréal visait le réaménagement des trois voies de circulation du boulevard Saint-Laurent sous le viaduc ferroviaire jusqu'à l'intersection avec la rue de Bellechasse (figure 3 et 4). Le passage sous le viaduc étant peu propice à la circulation des cyclistes en sécurité (rétrécissement de la chaussée, faible visibilité), piétons et cyclistes se 
partageaient les deux étroits trottoirs. L'objectif du réaménagement était d'améliorer la sécurité des piétons et cyclistes en retirant une voie de circulation, en ajoutant une piste cyclable et des feux de circulation sur le boulevard Saint-Laurent pour compenser le manque de visibilité à l'intersection de la rue de Bellechasse, immédiatement à la sortie du viaduc.

La création de la piste cyclable à contresens de la rue Shaw, à Toronto, répondait aussi en partie au besoin de sécuriser les déplacements des cyclistes. Il existait déjà une piste cyclable sur cette rue à sens unique, un axe nord-sud très utilisé en raison de la faible densité de la circulation automobile et du fait que la rue croise plusieurs autres pistes cyclables dans la direction est-ouest. Pour ces mêmes raisons, des cyclistes empruntaient déjà la rue vers le nord, malgré l'interdiction. Aménager la piste cyclable pour qu'elle soit utilisable dans les deux directions contribuait donc à leur sécurité. Enfin, sur la rue commerçante Roncesvalles, la modernisation des rails et des voitures $\mathrm{du}$ tramway offrait une occasion de repenser la cohabitation entre les usagers du transport en commun - qui sont aussi des piétons - et les automobilistes (figure 1). Des saillies de trottoirs ont été créées pour faciliter l'embarquement et le débarquement du tramway, sans possibilité d'entrer en collision avec les voitures qui circulent dans la rue. Plus tard dans le processus s'est ajoutée la préoccupation de repenser la cohabitation entre les usagers du transport en commun et les cyclistes afin de minimiser les risques de collision.

Figures 3 : New configurations of Saint-Laurent Boulevard towards Bellechasse Street in Montreal and of Roncesvalles Street in Toronto

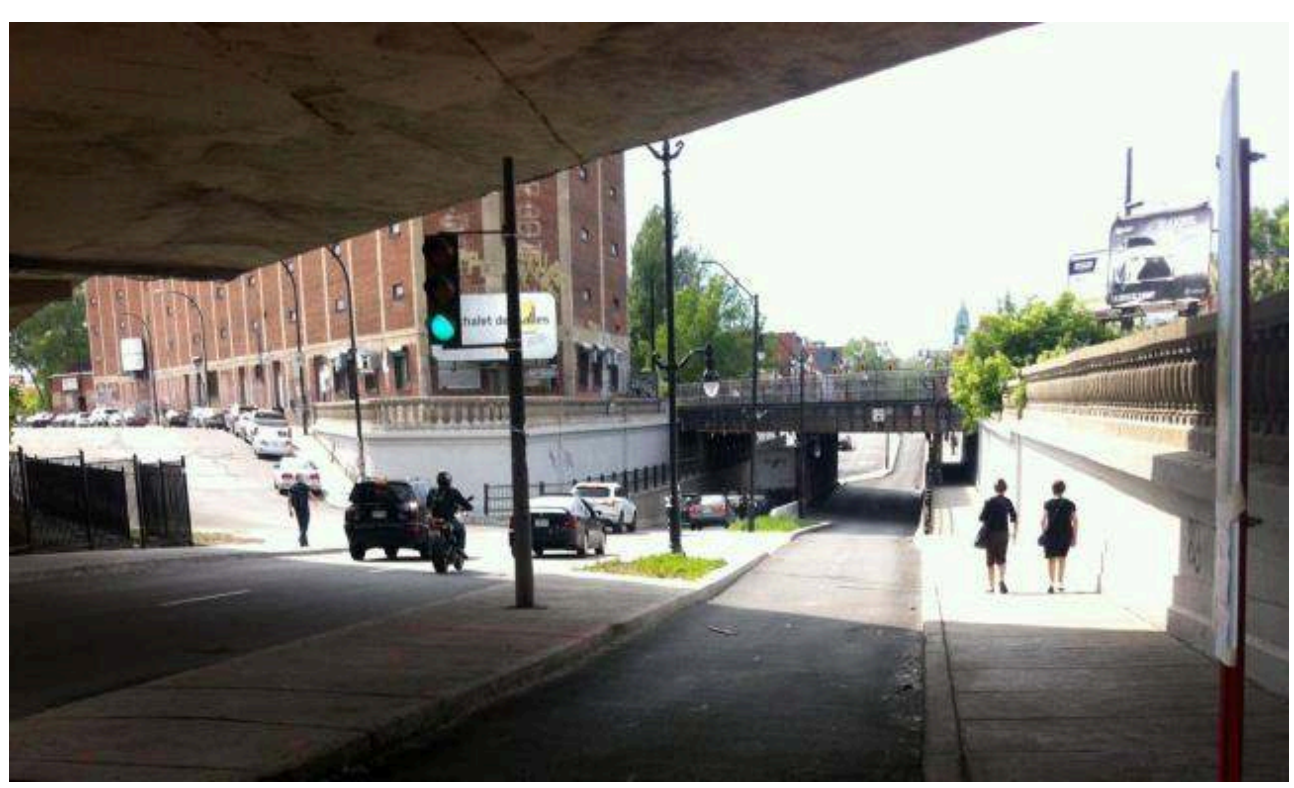

Photo : Nina Escoute, 2015 
Figures 4 : New configurations of Saint-Laurent Boulevard towards Bellechasse Street in Montreal and of Roncesvalles Street in Toronto

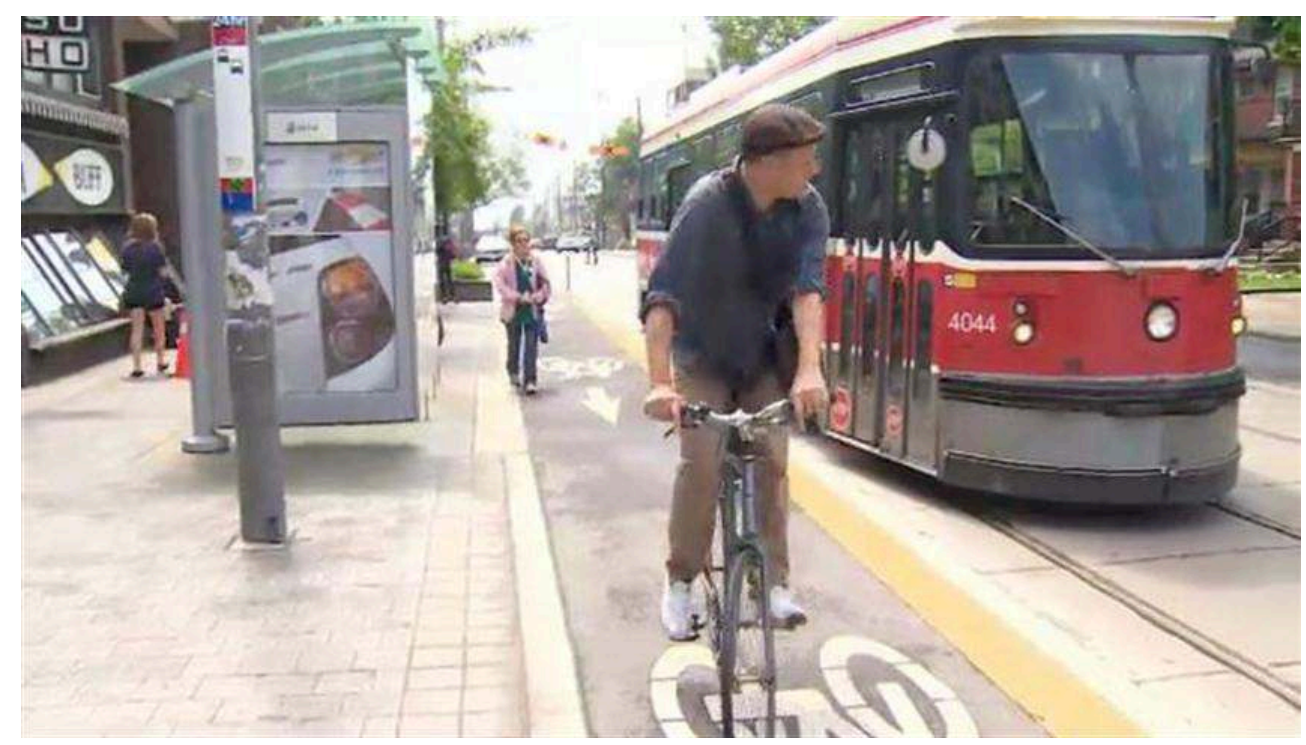

Photo : http://thumbnails.cbc.ca?

Si la santé est bien au cœur des justifications de ces initiatives spécifiques, elle n'en est pas la seule, ni même nécessairement la principale. Au souci de sécurité, s'ajoute la préoccupation de maintenir ou d'accroître la fluidité des déplacements pour tous les usagers, en particulier pour les cyclistes, dans les cas de l'intersection Saint-Laurent Bellechasse et de la piste cyclable à contresens de la rue Shaw. C'est la fonction « transport » du transport actif qui est invoquée ici. Dans les cas du Quartier Vert ParcExtension et de la rue commerçante Roncesvalles, l'enjeu était aussi d'accroître la convivialité des espaces, donc une dimension de bien-être dans la façon d'occuper l'espace public urbain. Enfin, on constate que dans deux des initiatives, la préoccupation de sécurisation des déplacements est subordonnée à des besoins de modernisation des infrastructures. Le réaménagement de l'intersection Saint-Laurent / Bellechasse n'a pu être entrepris que lorsque les travaux souterrains sur les réseaux d'aqueduc, d'égouts et d'électricité ont débuté. La revitalisation de la rue Roncesvalles a eu pour point de départ la volonté de la société de transport en commun de moderniser les rails pour accommoder les nouvelles voitures du tramway. La sécurisation des déplacements des usagers du tramway a été pensée en conséquence de cette modernisation, et non l'inverse.

\section{Les acteurs de santé publique, généralement absents de la mise en œuvre des initiatives étudiées}

Dans trois des quatre initiatives étudiées, les acteurs de santé publique ne sont nullement intervenus dans la décision de retenir et dans la mise en œuvre de ces projets concrets. Dans ces trois initiatives, les acteurs principaux étaient les élus et fonctionnaires des services de transport et des services techniques de la ville, les groupes de piétons et cyclistes, les groupes environnementaux concernés par le transport actif, les associations de commerçants, des citoyens et, dans quelques cas, des journalistes. 
40 significative - que dans le projet Quartier Vert Parc-Extension. Cette intervention est d'abord financière, car le projet Quartier Vert Actif et en Santé (QVAS) dans lequel s'est inscrite la démarche citoyenne de Parc-Extension est financée par deux organisations de santé publique, l'Agence de santé publique du Canada et la fondation Québec en Forme. Cette dernière est un organisme de santé publique qui résulte du partenariat pour la promotion de saines habitudes de vie entre le ministère de la Santé et des Services sociaux et la Fondation Lucie et André Chagnon. Dans le cadre du programme Quartier 21, la DSP de Montréal a soutenu financièrement des actions émanant de la démarche QVAS à Parc-Extension entre 2011 et 2014. Les acteurs de santé publique sont aussi intervenus dans le contenu de l'initiative par du mentorat et par la transmission de données. Pendant la démarche, les acteurs locaux ont bénéficié du soutien d'une conseillère de Québec en forme pour s'assurer que les propositions amenées permettraient de voir des projets et des actions concrètes et pérennes dans le quartier. La Coalition québécoise sur la problématique du poids a également contribué au développement des QVAS étant donné le lien établi entre santé et activité physique, tandis que la DSP de Montréal a mis à disposition des acteurs locaux des données de sécurité pour la réalisation du portrait diagnostic sur le quartier (Montréal, représentante d'un groupe d'intérêt environnemental).

\section{Discussion}

La question qui a guidé cet article était : la santé publique est-elle un acteur majeur des politiques de transport actif à Montréal et à Toronto ? Pour y répondre, nous avons repéré le rôle des acteurs de santé publique ainsi que la référence aux valeurs et connaissances en santé dans le sous-système de la politique du transport actif. Il en ressort quatre résultats principaux. Premièrement, des arguments fondés sur les valeurs et connaissances de santé ont circulé au-delà de la communauté de santé publique. On les retrouve dans les documents officiels des politiques de transport actif des deux villes, dans les justifications des initiatives spécifiques de transformation de l'environnement bâti étudiées et dans les discours d'acteurs d'autres secteurs. Deuxièmement, les acteurs de santé publique déploient des efforts considérables pour produire et transmettre des connaissances sur les liens entre transport, aménagement urbain et santé; et pour prendre position sur des enjeux de politiques publiques touchant au transport actif. Ils sont pourtant très peu présents dans la mise en œuvre des initiatives spécifiques à l'étude. Troisièmement, leurs interactions sont plus poussées avec les autres acteurs du sous-système qui partagent des valeurs quant au transport actif, en particulier les organismes non-gouvernementaux. Quatrièmement, leurs interactions sont plus limitées avec les acteurs qui ne partagent que partiellement ces valeurs, comme les directions municipales des transports.

Ces résultats appellent un premier élément de discussion : comment expliquer le rôle moteur des acteurs de santé publique dans l'animation et la progression du débat public sur le transport actif? Tout d'abord, pour reprendre les catégories conceptuelles de l'ACF, deux événements externes au sous-système du transport actif concentrent l'attention des acteurs d'autres secteurs sur les liens entre santé et transport. La reconnaissance d'un problème environnemental conduit les acteurs de santé publique à s'intéresser au transport dans les années 1990 à Montréal et Toronto, c'est-à-dire la

Revue francophone sur la santé et les territoires, Mobilités Transports et Santé 
piètre qualité de l'air résultant notamment de la pollution générée par les transports motorisés. Le second événement externe est en fait partagé entre les sous-systèmes du transport et de la santé publique puisque c'est la prise de conscience du nombre de blessures et de décès de cyclistes et de piétons lors de collisions avec des camions ou des automobiles. Ces événements établissent un contexte qui rend plus légitime le regard de la santé sur le transport actif, aux yeux des acteurs des autres secteurs. La deuxième piste d'explication du rôle moteur des acteurs de santé publique dans l'émergence des politiques de transport actif renvoie à leurs capacités de recherche et de construction de partenariat. Tant la DSP de Montréal que Toronto Public Health collaborent avec des universitaires et surtout emploient des chercheurs pour produire des données. Ces derniers sont généralement impliqués dans la transmission de leurs données par le dépôt de mémoires de consultation ou par des collaborations avec d'autres acteurs. De plus, d'autres employés ont pour mission d'établir et d'entretenir des partenariats avec des organismes non-gouvernementaux. Enfin, la compétence et la capacité budgétaire - un paramètre relativement stable, dans les termes de l'ACF accordée aux acteurs de santé publique pour soutenir financièrement les actions d'organismes non-gouvernementaux sur les déterminants sociaux de la santé contribuent à expliquer comment ils peuvent soutenir des initiatives favorables au développement du transport actif, comme le Quartier Vert Parc-Extension étudié ici.

Nos résultats soulignent toutefois les limites de l'influence des acteurs de santé publique sur les acteurs municipaux qui ont la maîtrise de l'agenda et de la capacité d'action en transport actif, ainsi que leur présence limitée dans la mise en œuvre des politiques de transport actif. Comment expliquer ce paradoxe apparent entre la forte présence des acteurs de santé publique dans l'émergence du transport actif et sa présence limitée dans la mise en œuvre ? Le premier élément d'explication tient à la structure constitutionnelle, l'un des paramètres relativement stables de l'ACF. Le choix des cas comparés permettait de tester l'hypothèse selon laquelle une direction de santé publique intégrée à l'administration municipale (Toronto) aurait une plus grande capacité d'influence sur cette dernière qu'une direction de santé publique extérieure à l'administration municipale (Montréal). Les résultats de la comparaison montrent que ce n'est pas le cas puisque tant Toronto Public Health que la DSP de Montréal font face à des critiques de leur implication en transport actif et n'interviennent que très peu dans la mise en œuvre. Le partage de compétences entre secteurs d'action publique (transport et santé publique) ressort comme la composante de la structure constitutionnelle la plus importante pour expliquer ces limites. En effet, comme le souligne une interlocutrice, les acteurs de santé publique n'ont pas les moyens opérationnels pour concrétiser leurs propositions (Toronto, acteurs de santé publique), implanter des pistes cyclables, construire des saillies de trottoir ou limiter la vitesse de circulation automobile. De plus, le partage formel des compétences est renforcé par la persistance du paradigme dominant en matière de transport, centré sur le transport routier et la compétence des ingénieurs en transport (Paulhiac \& Kaufmann, 2006). Au contraire, les interventions des acteurs de santé publique relèvent d'un paradigme de santé globale qui légitime des interventions sur les déterminants sociaux de la santé, c'est-à-dire dans les champs de compétence qui relèvent traditionnellement d'autres secteurs, incluant le système de transport. Cette conception de la santé conduit les acteurs de santé publique à sortir de leurs champs habituels d'intervention, ce que contestent notamment les acteurs des transports. Bien que ces deux paradigmes se 
rejoignent sur certaines questions - comme le montre l'importance accordée à la sécurité des déplacements - ils sont loin encore d'être intégrés.

Une des limites de notre recherche concerne le choix des initiatives spécifiques étudiées puisque toutes portent sur des transformations de l'environnement bâti en milieu micro-local. Ce type d'intervention relève entièrement de la responsabilité des municipalités, en particulier des élus et des directions des transports, de l'aménagement urbain et des travaux publics. Comme nous l'avons vu dans cet article, la concrétisation des initiatives est donc dépendante des valeurs de ces acteurs et de leurs rapports de pouvoir avec les autres acteurs du sous-système local et micro-local. Si nous avions retenu des interventions de modification des comportements (par exemple, une campagne visant à encourager les déplacements à vélo) ou des interventions nécessitant des changements réglementaires à l'échelle municipale (par exemple, pour permettre aux piétons de traverser les intersections en diagonale) ou provinciale (par exemple, des modifications au code de la route pour faciliter et sécuriser les déplacements à vélo), l'élaboration et la mise en œuvre des initiatives auraient fait intervenir des acteurs et des contraintes institutionnelles différents de ce que nous avons observé, modifiant ainsi le rôle des acteurs de santé publique. De même, si nous avions étudié des interventions de modification des comportements, l'expertise des acteurs de santé publique en matière de campagnes de prévention aurait pu leur conférer une capacité d'action plus importante, parallèlement à celle des acteurs provinciaux ou municipaux responsables des transports.

\section{Conclusion}

Cette recherche montre que les acteurs de santé publique concentrent leur travail sur les connaissances et les valeurs liant transport, urbanisme et santé, contribuant à faire évoluer le paradigme dominant de la politique de transport. En contrepoint à l'image d'une santé publique déconnectée de la dimension politique de l'action publique, nos résultats montrent plutôt une implication des acteurs de santé publique qui accompagnent la transmission des connaissances qu'ils produisent par des interactions avec les autres acteurs municipaux et par des collaborations avec des organismes nongouvernementaux. Ces interactions reposent sur divers points d'entrée (la sécurité des déplacements, la qualité de l'air, la prévalence de la sédentarité et de l'obésité), ce qui permet d'établir des alliances avec des acteurs spécialisés en transport comme en environnement ou en activité physique qui, eux, pourront faire circuler ces connaissances et ces valeurs par d'autres canaux. Malgré tout, la mise en œuvre des politiques de transport actif demeure la chasse-gardée des professionnels des secteurs "directement» concernés, en l'occurrence des ingénieurs en transport et des urbanistes.

En conclusion, les résultats présentés suggèrent qu'il n'est pas suffisant que des connaissances et des valeurs de santé soient incluses dans les plans officiels qui formalisent les objectifs des politiques de transport actif. Les règles constitutionnelles qui encadrent le partage de compétences entre secteurs, la conception des secteurs comme étant associés à une profession dominante et à un paradigme dominant limitent la capacité des acteurs de santé publique à faire en sorte que les politiques de transport actif deviennent encore plus favorables à la santé. Qu'est-ce que les acteurs de santé publique pourraient accomplir de plus s'ils étaient impliqués dans la mise en œuvre des 
politiques de transport actif, c'est-à-dire dès la définition des modalités d'action ? Considérant les liens entre le développement d'infrastructures cyclables et la gentrification (Flanagan, Lachapelle, \& El-Geneidy, 2016; Stehlin, 2015) ainsi que les inégalités sociales dans la pratique des déplacements actifs (Fuller \& Winters, 2017), nous suggérons que leur rôle pourrait être de porter la préoccupation pour les inégalités sociales et territoriales de santé en lien avec la sélection des rues ou des quartiers qui reçoivent les investissements ou avec les types d'aménagements de transport actif et leurs effets sur les inégalités sociales. Certes, l'effet maximal sur les inégalités sociales de santé se mesure à l'échelle des États, en réponse à des politiques redistributives notamment (Bambra et al., 2010 ; Navarro \& Shi, 2001). Cependant, les espaces locaux ont une influence sur les habitudes et les trajectoires de vie de leurs résidants (Bernard et al., 2007). Des transformations de l'environnement bâti local peuvent donc accentuer des écarts d'état de santé entre des populations plus ou moins favorisées, Prendre en compte les effets sur les populations vulnérables de multiples interventions de transport actif dès leur conception pourrait alors contribuer à assurer la cohérence entre des objectifs généraux de politique publique et les interventions qui les concrétisent. Autrement dit, sans se substituer aux autres professionnels concernés, la consultation plus systématique des acteurs de santé publique lors de la définition des modalités d'action visant à agir sur l'environnement bâti pourrait établir une continuité entre la préoccupation pour la santé exprimée dans les plans officiels et la contribution spécifique de différentes modalités d'intervention à la santé publique ou à la réduction des inégalités sociales de santé. La participation des acteurs de santé publique à la mise en œuvre permettrait donc de poursuivre le dialogue intersectoriel initié lors de l'émergence des politiques de transport actif, de considérer d'autres aspects des liens entre transport et santé, au-delà de la sécurité. Pour y parvenir, il y a encore un travail d'apprentissage à réaliser pour développer un fort consensus quant à l'intérêt collectif pour les politiques de transport actif.

\section{BIBLIOGRAPHIE}

Bambra, C., Gibson, M., Sowden, A., Wright, K., Whitehead, M., \& Petticrew, M. (2010). Tackling the wider social determinants of health and health inequalities: evidence from systematic reviews. Journal of Epidemiology and Community Health, 64 (4), 284-291.

Bernard, P., Charafeddine, R., Frohlich, K. L., Daniel, M., Kestens, Y., \& Potvin, L. (2007). Health inequalities and place: a theoretical conception of neighbourhood. Social Science \& Medicine, 65, 1839-1852.

Boudreau, J.-A., Hamel, P., Jouve, B., \& Keil, R. (2006). Comparing metropolitan governance: The cases of Montreal and Toronto. Progress in Planning, 66 (1), 7-59. doi:10.1016/j.progress.2006.07.005

Cervero, R., Sarmiento, O. L., Jacoby, E., Gomez, L. F., \& Neiman, A. (2009). Influences of Built Environments on Walking and Cycling: Lessons from Bogotá. International Journal of Sustainable Transportation, 3 (4), 203-226. doi:10.1080/15568310802178314 
City of Toronto. (2001). Toronto Bike Plan. Toronto: City of Toronto.

City of Toronto. (2002). Toronto Pedestrian Charter. Toronto.

City of Toronto. (2009). Toronto Walking Strategy Document

City of Toronto. (2014). Chapter 2 Shaping the City. Toronto Official Plan. Toronto: City of Toronto.

City of Toronto. (2019). Network Status. Retrieved from https://www.toronto.ca/servicespayments/streets-parking-transportation/cycling-in-toronto/cycle-track-projects/networkstatus/

Clavier, C., \& Gagnon, F. (2013). L'action intersectorielle en santé publique ou lorsque les institutions, les intérêts et les idées entrent en jeu. La Revue de l'innovation : La Revue de l'innovation dans le secteur public, 18 (2), article 2.

Dale, D. (2012, April 24, 2012). Lower Toronto speed limits by 10 to $20 \mathrm{~km} / \mathrm{h}$ to protect pedestrians, chief medical o!cer says. The Toronto Star. Retrieved from https://www.thestar.com/ news/city_hall/2012/04/24/

lower_toronto_speed...y_10_to_20_kmh_to_protect_pedestrians_chief_medical_officer_says.html de Leeuw, E. (2017). Engagement of Sectors Other than Health in Integrated Health Governance, Policy, and Action. Annual Review of Public Health, 38 (1), 329-349. doi:10.1146/annurevpublhealth-031816-044309

DeLeon, P. (1999). The stages approach to the policy process: what has it done? Where is it going? In P. A. Sabatier (Ed.), Theories of the Policy Process. Boulder, CO: Westview.

Direction de santé publique. (2006). Le transport urbain, une question de santé. Montréal: Agence de la santé et des services sociaux de Montréal.

Feuillet, T., Salze, P., Charreire, H., Menai, M., Enaux, C., Perchoux, C., ... Oppert, J.-M. (2016). Built environment in local relation with walking: Why here and not there? Journal of Transport \& Health, 3 (4), 500-512. doi:http://doi.org/10.1016/j.jth.2015.12.004

Flanagan, E., Lachapelle, U., \& El-Geneidy, A. (2016). Riding tandem: Does cycling infrastructure investment mirror gentrification and privilege in Portland, OR and Chicago, IL? Research in Transportation Economics, 60, 14-24. doi:https://doi.org/10.1016/j.retrec.2016.07.027

Frank, L., Giles-Corti, B., \& Ewing, R. (2016). The influence of the built environment on transport and health. Journal of Transport \& Health, 3 (4), 423-425. doi:http://doi.org/10.1016/j.jth.

2016.11.004

Fuller, D., \& Winters, M. (2017). Income inequalities in Bike Score and bicycling to work in Canada. Journal of Transport \& Health, 7, 264-268. doi:https://doi.org/10.1016/j.jth.2017.09.005

Giles-Corti, B., Foster, S., Shilton, T., \& Falconer, R. (2010). The co-benefits for health of investing in active transportation. N S W Public Health Bull, 21 (5-6), 122-127. doi:10.1071/nb10027

Hassenteufel, P. (2011). Sociologie politique: l'action publique (2ème édition). Paris: Armand-Colin.

Horak, M. (2012). Multilevel governance in Toronto: Success and failure in Canada's largest city. In M. Horak \& R. Young (Eds.), Sites of governance. Multilevel governance and policy-making in Canada's big cities (pp. 228-261). Montreal \& Kingston: McGill-Queen's University Press.

Litman, T. (2003). Active transportation policy issues. National Roundtable on Active Transportation, 13.

Marmot, M., \& Wilkinson, R. (Eds.). (2006). Social Determinants of Health 2nd Edition. Oxford: Oxford University Press. 
Morency, P., Archambault, J., Cloutier, M.-S., Tremblay, M., Plante, C., \& Dubé, A. S. (2013). Sécurité des piétons en milieu urbain: enquête sur les aménagements routiers aux intersections. Retrieved from Montréal:

Navarro, V., \& Shi, L. (2001). The political context of social inequalities and health. Social Science \& Medicine, 52 (3), 481-491.

Paquin, S., \& Dubé, A.-S. (2011). La carte conceptuelle du transport actif urbain. Cahiers de géographie du Québec, 55 (156), 399-428.

Paulhiac, F., \& Kaufmann, V. (2006). Transports urbains à Montréal : évolutions des référentiels et enjeux d'une politique durable. Revue d'Économie Régionale \& Urbaine (1), 49-80.

Reynolds, C. (2010). Active transportation in urban areas exploring health benefits and risks (9780981264684 0981264689). Retrieved from Vancouver, B.C.

Rider, D. (2012, May 2, 2012 ). Toronto's medical o!cer unfazed by Ford brothers' criticism. Toronto Star. Retrieved from https://www.thestar.com/news/city_hall/2012/05/02/ torontos_medical_officer_unfazed_by_ford_brot hers_criticism.html

Sabatier, P. A., \& Weible, C. M. (2007). The Advocacy Coalition Framework: Innovations and Clarifications. In P. A. Sabatier (Ed.), Theories of the Policy Process. 2nd Edition (pp. 189-220). Boulder, Co: Westview Press.

Sartori, G. (1991). Comparing and miscomparing. Journal of Theoretical Politics, 3 (3), 243-257.

Statistics Canada. (2017). Commuters using sustainable transportation in census metropolitan areas. Census of population, 2016. Retrieved from Ottawa.

Stehlin, J. (2015). Cycles of Investment: Bicycle Infrastructure, Gentrification, and the Restructuring of the San Francisco Bay Area. Environment and Planning A: Economy and Space, 47 (1), 121-137. doi:10.1068/a130098p

Toronto Cycling. (2012). Mapping cycling behaviour in Toronto. Retrieved from Toronto.

Toronto Public Health. (2011). Healthy Toronto by Design. Retrieved from Toronto, Ontario.

Toronto Public Health. (2012). Road to Health: Improving Walking and Cycling in Toronto. Toronto: Toronto Public Health, Healthy Public Policy Directorate.

Toronto Public Health. (2013). Next Stop Health: Transit Access and Health Inequities in Toronto. Toronto: Toronto Public Health.

Toronto Public Health. (2014). Active City: Designing for Health.

Vélo Québec. (2015). L'état du vélo au Québec en 2015. Montréal: Vélo Québec Association.

Ville de Montréal. (2008). Plan de transport de 2008. Montréal: Ville de Montréal.

Weible, C. M., Sabatier, P. A., Jenkins-Smith, H. C., Nohrstedt, D., Henry, A. D., \& deLeon, P. (2011). A Quarter Century of the Advocacy Coalition Framework: An Introduction to the Special Issue. Policy Studies Journal, 39 (3), 349-360. doi:10.1111/j.1541-0072.2011.00412.x

\section{NOTES}

1. Référence complète : http://thumbnails.cbc.ca/maven_legacy/thumbnails/944/483/ Bikes-Matteis_852x480_2388545144.jpg 


\section{NOTES DE FIN}

i. Les extraits d'entretiens réalisés à Toronto ont été conservés en anglais afin de ne pas perdre de sens lors de la traduction.

\section{RÉSUMÉS}

Les politiques urbaines qui encouragent les déplacements à pied et à vélo sont reconnues pour être potentiellement favorables à la santé. Pourtant, la recherche s'est peu intéressée au rôle des acteurs de santé publique dans l'émergence et la mise en œuvre des politiques de transport actif. L'article questionne ce rôle des acteurs de santé publique à partir des résultats d'une recherche comparative à Montréal et Toronto (1970-2016). Suivant le cadre théorique des coalitions de cause et à partir d'une analyse documentaire et d'entretiens semi-directifs, nous étudions les interactions des acteurs de santé publique et les valeurs de santé dans la politique. Nos résultats montrent le paradoxe de la présence des acteurs et des valeurs de santé dans l'émergence des politiques de transport actif, et de leur relative absence dans la mise en œuvre. Les résultats ouvrent une réflexion sur la contribution des acteurs de santé publique aux politiques urbaines.

Urban policies that encourage walking or cycling to work are widely considered as healthy public policies given the benefits of increased physical activity, reduced traffic congestion and reduced air pollution. The difficulty for public health actors is that action to promote walking or cycling is largely outside their reach, for instance : building walking and cycling infrastructure (broader sidewalks, cycling lanes, and so on), improving access to public transit, or changing bylaws on maximum speed limits. These actions fall under the responsibility of transportation and urban planning sectors. In other words, creating healthy public policies requires that public health actors partner with actors from other sectors. In doing so, they are likely to come up against « politics and power games " (de Leeuw, 2017, p. 344). This paper explores what public health actors do to integrate a health perspective into urban policies for active transportation. As policy instruments fall under the responsibility of other sectors, what do they do? Are they limited to advocacy efforts? And how can these policy instruments be implemented when these other sectors are primarily concerned with something other than health? To answer these questions, this paper examines the role of public health actors in the process of leading two major Canadian cities, Montreal and Toronto, to develop active transportation policies (the policy emergence phase) and to implement these policies, including specific initiatives to change the built environment for active transportation (the implementation phase). We observe how public health actors, and in particular the local public health authorities, interact with actors from other sectors concerned with active transportation. We also consider how health knowledge and values are used in the legitimisation of active transportation policies. This focus on interactions and values stems from our use of an advocacy coalition framework (Sabatier \& Weible, 2007). The methods include documentary analysis (official plans and documents related to active transportation) and semi-structured interviews (Montreal=20, Toronto=20) with key actors from the active transportation policy subsystem (in particular: municipal councillors and employees; public health actors, especially from local public health agencies; representatives from interest groups involved with active transportation).

There are four main results from this study. Firstly, arguments for active transportation based on health benefits have circulated beyond the community of public health actors. Such arguments are found in the official plans stating the objectives of both cities' active transportation policies; 
in rationales justifying the specific initiatives under study; and in the discourses of actors from other sectors. Secondly, public health actors spend considerable time and effort in the production and diffusion of knowledge regarding the intersections between transportation, urban planning and health. Based on this knowledge, they also adopt public positions on policy issues regarding active transportation. They are, however, largely absent from the implementation phase of the specific active transportation initiatives under study here (figure 1). Third, public health actors engage with actors from other policy sectors that share their values and vision regarding active transportation policies. This is especially true of their interactions with non-governmental organisations and university researchers. Lastly, it is more difficult for them to establish interactions with actors that do not share their values and visions regarding preferred policies, especially with municipal transportation services.

In conclusion, our results suggest that the inclusion of health knowledge and values into official plans that state the objectives of active transportation policies is not sufficient to determine that health has become part of active transportation policies. Constitutional rules that define how responsibilities are shared between sectors, and the definition of a policy sector by a dominant profession and a dominant policy paradigm, limit the extent to which public health actors may shape active transportation policies so that they become healthier urban policies.

\section{INDEX}

Mots-clés : politiques locales, transports, santé publique, politiques urbaines

\section{AUTEURS}

\section{CAROLE CLAVIER}

Professeure, Département de science politique, Université du Québec à Montréal

\section{FRANCE GAGNON}

Professeure, École des sciences de l'administration, TÉLUQ

\section{SOPHIE PAQUIN}

Professeure, Département d'études urbaines et touristiques, ESG UQAM

\section{KATIE HAYES}

Doctorante, Dalla Lana School of Public Health, Université de Toronto

\section{BLAKE POLAND}

Professeur, Dalla Lana School of Public Health, Université de Toronto

BETH SAVAN

Sr. Lecturer (Emeritus), School of the Environment, Université de Toronto

\section{NINA ESCOUTE}

Assistante de recherche, Département de science politique, UQAM 